\title{
Correlation between Expression Differences of Epithelial-Mesenchymal Transition (EMT) in Cholangiocarcinoma Tissue
}

\author{
Qi Liu1, Xiang Rao', Haixiang Cai' ${ }^{1}$ Juncheng Guo ${ }^{2 *}$ \\ ${ }^{1}$ The Fourth People's Hospital of Haikou, Haikou, China \\ ${ }^{2}$ Hainan General Hospital, Haikou, China \\ Email: ^g2002m@163.com
}

How to cite this paper: Liu, Q., Rao, X., Cai, H.X. and Guo, J.C. (2021) Correlation between Expression Differences of Epithelial-Mesenchymal Transition (EMT) in Cholangiocarcinoma Tissue. International Journal of Clinical Medicine, 12, 328-341. https://doi.org/10.4236/ijcm.2021.128029

Received: July 23, 2021

Accepted: August 10, 2021

Published: August 13, 2021

Copyright $\odot 2021$ by author(s) and Scientific Research Publishing Inc. This work is licensed under the Creative Commons Attribution International License (CC BY 4.0).

http://creativecommons.org/licenses/by/4.0/

\begin{abstract}
Background: By studying the expression of epithelial-mesenchymal transition regulators in cholangiocarcinoma and intrahepatic duct stones, the correlation between the expression of epithelial-mesenchymal transition regulators and cholangiocarcinoma was revealed. Objective: The objective is to investigate the correlation between the expression of epithelial-mesenchymal transition (EC) regulatory factors and cholangiocarcinoma in patients with intrahepatic duct stones and cholangiocarcinoma, to investigate the relationship between clinicopathological features and prognosis, and to observe the expression of molecular markers of epithelial-mesenchymal transition (EMT) in intrahepatic duct stones and bile duct carcinoma. Methods: Twenty cases of primary cholangiocarcinoma, 20 cases of intrahepatic cholangiolithiasis complicated with cholangiocarcinoma, and 20 cases of intrahepatic cholangiolithiasis specimens were collected from the Fourth People's Hospital and the friendly medical unit of Haikou. Immunohistochemistry was used to detect the expression differences of EMT-related molecular markers Twisit1, Twisit2, E-cadherin, $\mathrm{N}$-cadherin, and Vimentin in paraffin sections of normal intrahepatic bile duct tissues and patients with intrahepatic duct stones and cholangiocarcinoma. Results: Immunohistochemical staining revealed epithelial-mesenchymal transition (EMT) in intrahepatic cholangiocarcinoma tissue, intrahepatic cholangiolithiasis with cholangiocarcinoma, intrahepatic cholangiolithiasis with normal intrahepatic cholangiolithiasis, such as Sit1, Twisit2, E-cadherin, N-cadherin, and Vimentin proteins were different. The expression of E-cadherin was decreased in cholangiocarcinoma tissue and intrahepatic cholangiolithiocarcinoma combined with cholangiocarcinoma $(\mathrm{P}$ $<0.05)$, while the expression of N-cadherin and Vimentin was up-regulated $(\mathrm{P}<0.05)$. The expression of Twisit1 and Twisit2 had no difference $(\mathrm{P}>$
\end{abstract}


0.05). There was no difference in the expression of intrahepatic bile duct stones and EMT $(\mathrm{P}>0.05)$. Conclusion: The expression of E-cadherin, the molecular marker of EMT, was down-regulated, while the expression of $\mathrm{N}$-cadherin and Vimentin was up-regulated. Age, gender, depth of tumor invasion, degree of tumor differentiation and lymph node metastasis were correlated with the expression of EMT in intrahepatic cholangiocarcinoma.

\section{Keywords}

Intrahepatic Bile Duct Stones, Hepatobiliary Cell Carcinoma, Organizations, EMT. Immunohistochemical

\section{Brief Introduction}

Intravenous cholangiocarcinoma (ICC) usually refers to the primary tumors in the hepatic duct, also known as peripheral bile duct or bile duct cell carcinoma, which originates from the secondary and above bile ducts in the liver. Malignant tumor is a serious threat to human health, and its mortality rate ranks first in both the world and China. According to the Global Cancer Statistics published by the World Health Organization in 2011 [1], Cholangiocarcinoma (CCA) originates from bile duct epithelial cells and is the most common malignant tumor in the liver and biliary system except bile duct carcinoma. According to different anatomical locations, cholangiocarcinoma can be divided into intrahepatic cholangiocarcinoma (IHCC), hilar cholangiocarcinoma Perihilar CCA (PHCC), and distal cholangiocarcinoma (DCC). Recent studies have reported that the incidence and mortality of cholangiocarcinoma are on the rise. Cholangiocarcinoma is aggressive and metastases early and more than $2 / 3$ of patients have lost the opportunity of surgery when diagnosed. Moreover, the effect of adjuvant therapy for cholangiocarcinoma still needs to be further verified. The median overall survival for patients with cholangiocarcinoma was only 15 months. Therefore, it is of great significance to further clarify the molecular mechanism of the occurrence and development of cholangiocarcinoma and to search for early diagnostic indicators and effective therapeutic targets of cholangiocarcinoma for improving the prognosis of patients. The etiology of cholangiocarcinoma is still unclear, but some relatively clear risk factors include primary sclerosing cholangitis [2], intrahepatic bile duct calculi [3], bile duct cyst, hepatic fluke [4], etc. Other possible risk factors include diabetes mellitus, HBV [5] and HCV virus infection [6]. However, the pathogenesis of intrahepatic cholangiocarcinoma has not yet been determined. With the continuous development of medical diagnostic techniques and the deepening of the understanding of this disease, considerable progress has been made in the study of the risk factors for its incidence, such as intrahepatic cholangiolithiasis, primary sclerosis cholangitis, congenital cholangitis cyst, etc. [7]. China is a country with a high incidence of intrahepatic bile duct stones, so intrahepatic bile duct stones are considered to be a common cause of intra- 
hepatic bile duct carcinoma in China. Studies have shown that about $10 \%$ of patients with intrahepatic bile duct stones may develop into intrahepatic bile duct carcinoma [8]. Hepatobiliary calculus with cholangiocarcinoma (HCWC) and hepatobiliary calculus associated with bile duct carcinoma was described. Many special factors above intrahepatic bile duct calculi-related bile duct carcinoma lead to its low early diagnosis rate, poor prognosis, high mortality rate, and even an increasing trend year by year. Therefore, more and more attention has been paid to the disease at home and abroad, and the research on the pathogenesis and prognostic factors will become an inevitable trend. Epithelial-mesenchymal transition (EMT) has increasingly become a hot research direction to explain the mechanism of tumor metastasis and invasion. The specific process of EMT includes loss of adhesion between cells, depolarization of cells, enhanced ability of cell invasion and migration, etc. The morphology of mesenchymal cells is conducive to the migration of tumor cells to distant organs and the maintenance of stem cell characteristics and is conducive to the transformation of tumor cells to other cell types in the initial and developmental stages of metastasis. However, the role and mechanism of EMT in cholangiocarcinoma are less studied. Therefore, it is of great value to further clarify the mechanism of EMT in cholangiocarcinoma for exploring its metastatic mechanism. Early diagnosis can significantly improve the therapeutic effect and prognosis of cholangiocarcinoma.

Unfortunately, at present, most patients with cholangiocarcinoma are diagnosed in the advanced stage and lose the best opportunity for surgical treatment. Therefore, the research on early cholangiocarcinoma and even precancerous lesions and the establishment of effective screening and diagnosis methods will play a decisive role in the prevention and control of cholangiocarcinoma. In the field of cholangiocarcinoma treatment, with the deepening of research, the treatment of cholangiocarcinoma is developing towards the direction of high pertinence and individualization, which is mainly determined by the molecular biological characteristics of cholangiocarcinoma cells. The individualized treatment of tumor requires the establishment of accurate and effective diagnostic indicators to group patients, so as to provide the basis for prognosis judgment and treatment program selection. Tumor molecular typing will play an important role in this field.

\section{Research Objects and Methods}

\subsection{Subjects}

Twenty patients with primary cholangiocarcinoma, 20 patients with intrahepatic cholangiolithiasis complicated with cholangiocarcinoma, and 20 patients with intrahepatic cholangiolithiasis were collected from the Fourth People's Hospital of Haikou during January 2020 to December 2020, and the case data of 20 paraffin pathological specimens were collected. Normal bile duct tissue (i.e., normal bile duct tissue $2 \mathrm{~cm}$ away from the margin of tumor or stone tissue) was used as control. 
Inclusion and exclusion criteria:

1) The patient was pathologically diagnosed as intrahepatic cholangiocarcinoma, and patients with mixed hepatocellular and cholangiocarcinoma were excluded.

2) The patient was the first to find ICC, and the patients with postoperative recurrence of ICC were excluded.

3) ICC patients treated with radical surgery were excluded from ICC patients treated with palliative surgery.

4) Hepatitis related ICC patients with metabolic syndrome, intrahepatic bile duct stones, schistosoma liver disease, alcoholism, primary sclerosing cholangitis or congenital intrahepatic bile duct cystic dilatation should be excluded, and those with hepatitis $\mathrm{C}$ infection should be considered.

Each patient in this study was approved by the Ethics Committee of the Fourth People's Hospital of Haikou City. This study was conducted in strict accordance with the Helsinki Declaration, and informed consent was signed. Paraffin white tablets for the experiment were prepared by the Department of Pathology (4 $\mu \mathrm{m} /$ tablet).

\subsection{Experimental Methods}

\subsubsection{Immunohistochemical Staining}

1) slice and bake: $4 \mu \mathrm{m}$ slice and bake at $60^{\circ} \mathrm{C}$ for 90 minutes.

2) Dewaxing and rehydrating: after the paraffin section is put into the bracket, it is immersed in xylene for 15 minutes, and then washed with distilled water, it is repeated again. Then the slices were soaked in 100\%, 95\%, 85\% and 75\% ethanol solutions for 5 minutes successively, and then rinsed thoroughly with distilled water repeatedly.

3) Heat repair antigen: using high temperature and high pressure method, the antigen repair solution was boiled in the pressure cooker and then put into the rinsed section, and then continued to heat and boil for 5 minutes. After cooling, the antigen repair solution was washed with PBS for 3 times, each time for 3 minutes.

4) Sealing: 3\% hydrogen peroxide was added to the section as the sealing solution and soaked at room temperature to inactivating endogenous peroxidase. After sealing for 10 minutes, the section was taken out and put on the scaffold. The section was washed with PBS for 3 times, 5 minutes each time, and then placed on the shaker.

5) Incubation of primary antibody: drop the properly diluted primary antibody and put it in a wet box at $4^{\circ} \mathrm{C}$ overnight. After removal, rewarm at room temperature at $37^{\circ} \mathrm{C}$ for 45 minutes, discard the primary antibody, and rinse with PBS for 3 times with at least 5 minutes for each rinse.

6) Incubation of the secondary antibody: drop appropriate secondary antibody to cover the specimen according to the instructions of the kit. Incubate at room temperature of $37^{\circ} \mathrm{C}$ for 60 minutes, rinse with $\mathrm{PBS}$ after discarding the 
secondary antibody, and repeat for 3 times.

7) $\mathrm{DAB}$ dyeing: $\mathrm{DAB}$ dyeing shall be performed according to the instructions of DAB kit. The real-time situation of dyeing shall be judged at any time. The dyeing time shall be determined according to the concentration of dyeing.

8) Re-staining: Drops of hematoxylin were added for nuclear re-staining. After 30 seconds of reaction, PBS was fully rinsed.

9) Dehydration sealing: the slices were placed in $75 \%, 85 \%, 95 \%$ and $100 \%$ ethanol solutions in turn and soaked for dehydration from low to high, for 2 minutes each time. After PBS rinsing, the slices were placed in xylene for $3 \mathrm{mi}-$ nutes and the excess xylene was erased. Drop neutral gum sealing piece, cover glass sealing piece, as for room temperature air drying pieces.

10) Put the air-dried wafer under a microscope for observation and interpretation.

\subsubsection{Observation and Analysis of the Results of Immunohistochemical Staining \\ Positioning results:}

E-cadherin was mainly expressed in the cell membrane, $\mathrm{N}$-cadherin was expressed in the cell membrane and cytoplasm, Twist 1 and Twist 2 were mainly expressed in the cytoplasm, and Vimentin was expressed in the cytoplasm.

Judgment criteria:

1) The brown-yellow particles in the cells were regarded as positive staining, and the cells were scored according to the degree of cell staining: 0 for no obvious staining, 1 for light yellow, 2 for dark yellow, and 3 for brown.

2) Randomly observe the sample area of each patient section, count 100 cells from each area, and then grade the specimen according to the ratio of positive cells to the total number of cells: When the percentage of positive stained bile duct cells in the total number of cells is less than $10 \%, 0$ marks, $10 \%-25 \%$ is 1 mark, $25 \%-50 \%$ is 2 marks, $50 \%-75 \%$ is 3 marks, and more than $75 \%$ is 4 marks. Calculate the product of cell staining depth score and cell number score. If the product is 0 , it is negative (-), $1-4$ is weak positive (+), $5-8$ is moderate positive $(++)$, and more than 9 is strong positive $(+++)$. Set \pm as (low) negative expression and $++/++$ as (high) positive expression. The results of all specimens were determined by three senior professional physicians in the Department of Pathology, and the counting data were averaged. After the data were collected and verified, the data were recorded and processed.

\subsubsection{Statistical Analysis}

The measurement data were expressed by means \pm standard deviation (SDS), and the independent sample $t$ test, $\chi^{2}$ test and correlation analysis were used for data analysis. Kaplan-Meier method was used to draw survival curves, and log-rank test was used to compare the differences. Statistical analysis was performed using SPSS 23.0 software (SPSS, Chicago, IL, USA), and the statistical analysis and mapping were performed in GraphPad Prism 6.0 software (San Diego, CA, USA). $\mathrm{P}<0.05$ was considered statistically significant. 


\section{Results}

\subsection{Intrahepatic Bile Duct Stones + Normal Tissues and the Expression of Molecular Markers Related to EMT}

There is no difference in the expression of molecular markers (see Figures 1(b)-(f)) related to intrahepatic bile duct stones + normal tissue and EMT(see Figure 1(a)). All images were under $40 \times 10$ microscope, and there was no difference in intrahepatic bile duct stones + normal tissue and EMT expression $(\mathrm{P}>0.05)$.

\subsection{E-Cadherin and the Expression of Normal Bile Duct Tissue and Hepatocholangiocarcinoma}

E-cadherin expression (normal bile duct tissue as shown in Figure 2(a) and hepatobiliary duct cell carcinoma as shown in Figure 2(b)). All images showed decreased E-cadherin expression under $40 \times 10$ microscopy.

Immunohistochemical results showed as shown in Figure 2, the positive expression of E-cadherin in cholangiocarcinoma tissues was 5 cases, accounting for $25 \%$ of the total, while the positive expression of E-cadherin in normal bile duct tissues was 16 cases, accounting for $80 \%$ of the total. The positive expression of E-cadherin in cholangiocarcinoma tissues was significantly down-regulated, as shown in Table $1(\mathrm{P}<0.01)$.

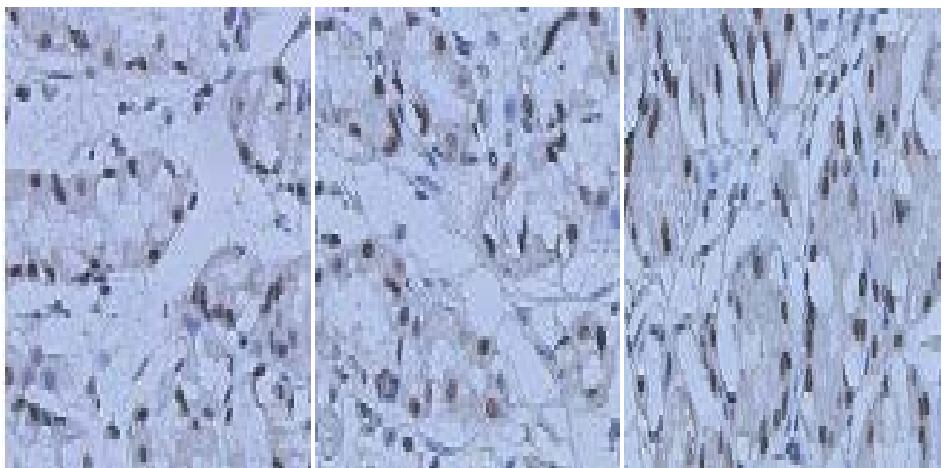

(a)

(b)

(c)

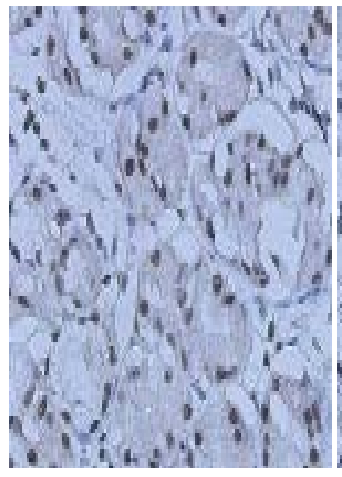

(d)

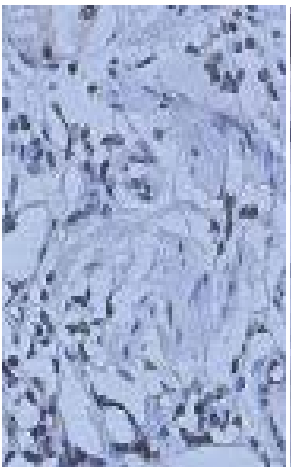

(e)

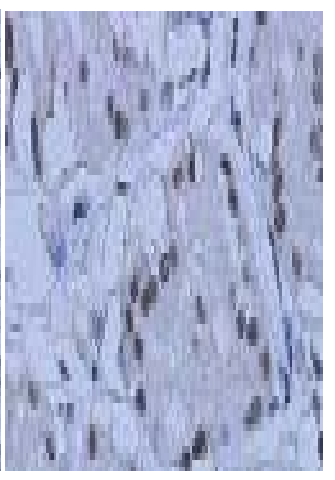

(f)

Figure 1. (a) Normal bile duct tissue; (b) Twisit1; (c) Twisit2; (d) E-cadhern; (e) N-cadhern; (f) Vimentin. 


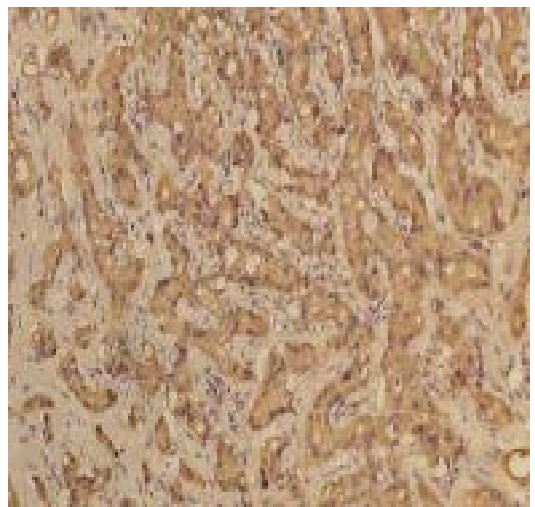

(a)

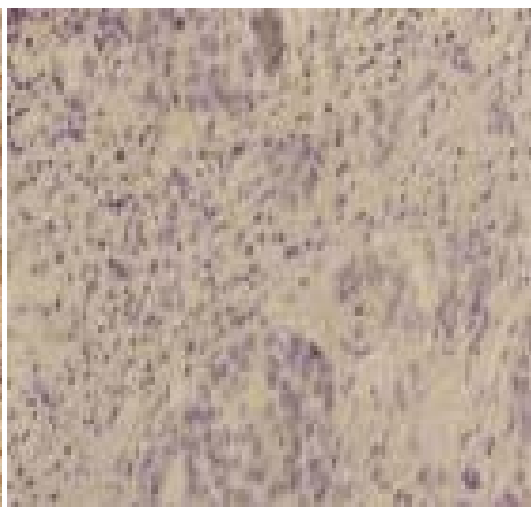

(b)

Figure 2. (a) Normal bile duct tissue; (b) Intrahepatic cholangiocarcinoma.

Table 1. Expression of E-cadherin in normal bile duct tissues and bile duct carcinoma tissues $(\mathrm{P}<0.01)$.

\begin{tabular}{cccc}
\hline Tissue types & $\mathrm{N}$ & $\begin{array}{c}\text { negative } \\
- \text { or }+\end{array}$ & $\begin{array}{c}\text { positive } \\
++ \text { or }+++\end{array}$ \\
\hline Normal bile duct tissue & $20(\%)$ & $14(70)$ & $6(30)$ \\
Intrahepatic cholangiocarcinoma & $20(\%)$ & $15(75)$ & $5(25)$ \\
\hline
\end{tabular}

\subsection{Expression of E-Cadherin and Intrahepatic Bile Duct Stones with Intrahepatic Cholangiocarcinoma}

E-cadherin expression (normal bile duct tissue as shown in Figure 3(a) and intrahepatic bile duct stones with intrahepatic bile duct carcinoma as shown in Figure 3(b)), resulting in decreased E-cadherin expression.

Immunohistochemical results showed as shown in Figure 3, the positive expression of E-cadherin in intrahepatic bile duct stones and intrahepatic bile duct carcinoma was 6 cases, accounting for 33.3\% of the total, while 15 cases, accounting for $75 \%$ of the total, were found in normal bile duct tissues. The positive expression of E-cadherin in intrahepatic bile duct stones and intrahepatic bile duct carcinoma was significantly down-regulated. See Table $2(\mathrm{P}<0.01)$.

\subsection{N-Cadherin and the Expression of Normal Bile Duct Tissue and Hepatocholangiocarcinoma}

Expression of N-cadherin (normal bile duct tissue see Figure 4(a) and hepatobiliary duct cell carcinoma see Figure 4(b)), resulting in elevated N-cadherin expression.

Immunohistochemical results showed as shown in Figure 4, the positive expression of $\mathrm{N}$-cadherin in cholangiocarcinoma tissues was 15 cases, accounting for $75 \%$ of the total, while the positive expression of $\mathrm{N}$-cadherin in normal bile duct tissues was 6 cases, accounting for $20 \%$ of the total. The positive expression of $\mathrm{N}$-cadherin in cholangiocarcinoma tissues was significantly up-regulated, as shown in Table $3(\mathrm{P}<0.01)$. 


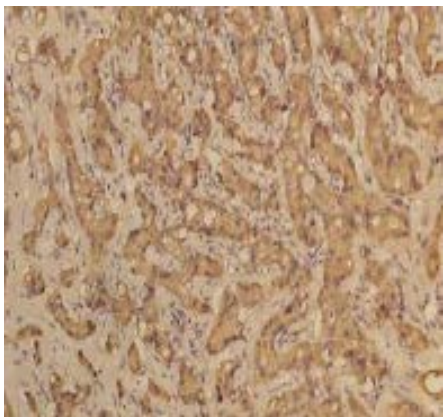

(a)

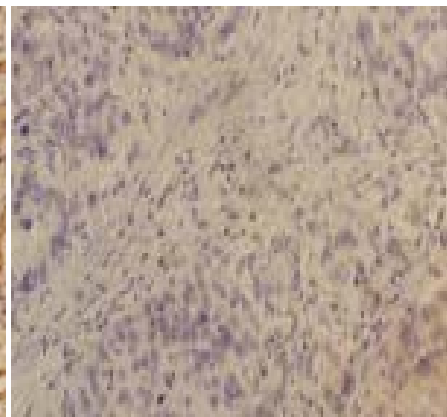

(b)

Figure 3. (a) Normal bile duct tissue; (b) Stone + intrahepatic cholangiocarcinoma.

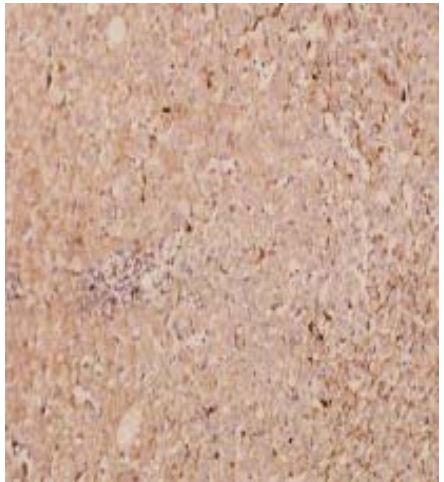

(a)

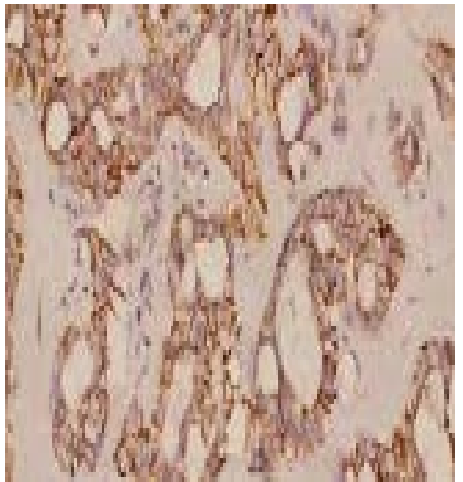

(b)

Figure 4. (a) Normal bile duct tissue; (b) Intrahepatic cholangiocarcinoma.

Table 2. Expression of E-cadherin in normal bile duct tissue, intrahepatic bile duct stones and intrahepatic bile duct carcinoma $(\mathrm{P}<0.01)$.

\begin{tabular}{cccc}
\hline Tissue types & N & $\begin{array}{c}\text { negative } \\
- \text { or }+\end{array}$ & $\begin{array}{c}\text { positive } \\
++ \text { or }+++\end{array}$ \\
\hline Normal bile duct tissue & $20(\%)$ & $5(25)$ & $15(75)$ \\
Stone + intrahepatic cholangiocarcinoma & $20(\%)$ & $14(70)$ & $6(30)$ \\
\hline
\end{tabular}

Table 3. Expression of $\mathrm{N}$-cadherin in normal bile duct tissues and bile duct carcinoma tissues $(\mathrm{P}<0.01)$.

\begin{tabular}{cccc}
\hline Tissue types & $\mathrm{N}$ & $\begin{array}{c}\text { negative } \\
- \text { or }+\end{array}$ & $\begin{array}{c}\text { positive } \\
++ \text { or }+++\end{array}$ \\
\hline Normal bile duct tissue & $20(\%)$ & $14(70)$ & $6(30)$ \\
Intrahepatic cholangiocarcinoma & $20(\%)$ & $5(25)$ & $15(75)$ \\
\hline
\end{tabular}

\subsection{N-Cadherin and the Expression of Intrahepatolithiasis + Intrahepatic Cholangiocarcinoma}

Expression of N-cadherin (normal bile duct tissue see Figure 5(a) and intrahepatic bile duct stones + intrahepatic bile duct carcinoma see Figure 5(b)), resulting in increased expression of $\mathrm{N}$-cadherin. 
Immunohistochemical results showed as shown in Figure 5, the positive expression of $\mathrm{N}$-cadherin in intrahepatic bile duct stones and intrahepatic bile duct carcinoma tissues was 15 cases, accounting for $75 \%$ of the total, while 5 cases in normal bile duct tissues, accounting for $25 \%$ of the total. The positive expression of $\mathrm{N}$-cadherin in intrahepatic bile duct stones and intrahepatic bile duct carcinoma tissues was significantly up-regulated. See Table $4(\mathrm{P}<0.01)$.

\subsection{Vimentin and the Expression of Orthohepatocholangiocarcinoma}

Vimentin expression in normal bile duct tissue see Figure 6(a) and hepatobiliary duct cell carcinoma see Figure 6(b), resulting in elevated Vimentin expression.

Immunohistochemical results showed as shown in Figure 6, the positive expression of $\mathrm{N}$-cadherin in cholangiocarcinoma tissues was 16 cases, accounting for $80 \%$ of the total, while the positive expression of $\mathrm{N}$-cadherin in normal bile duct tissues was 7 cases, accounting for $35 \%$ of the total. The positive expression of $\mathrm{N}$-cadherin in cholangiocarcinoma tissues was significantly up-regulated, as shown in Table $5(\mathrm{P}<0.01)$.

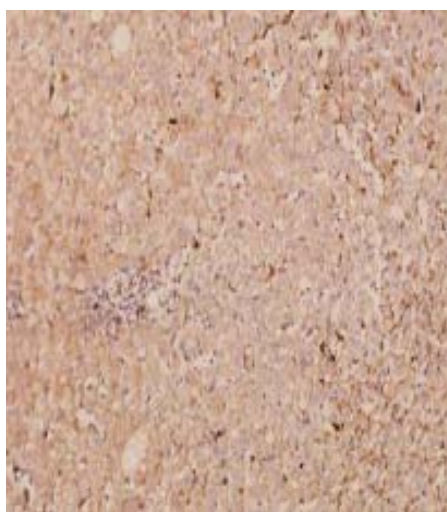

(a)

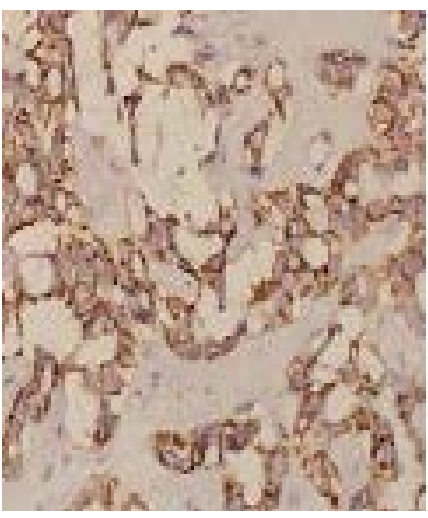

(b)

Figure 5. (a) Normal bile duct tissue; (b) Stone + intrahepatic cholangiocarcinoma.

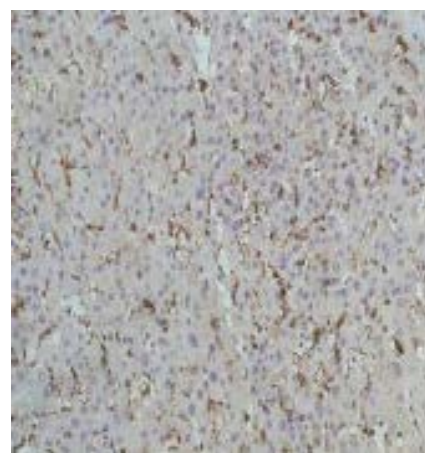

(a)

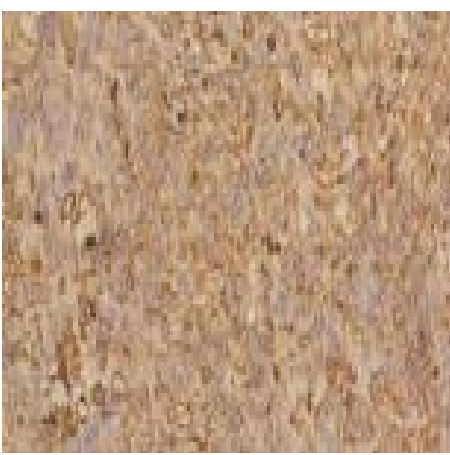

(b)

Figure 6. (a) Normal bile duct tissue; (b) Intrahepatic cholangiocarcinoma. 
Table 4. Expression of $\mathrm{N}$-cadherin in normal bile duct tissue, intrahepatic bile duct stones and intrahepatic bile duct carcinoma $(\mathrm{P}<0.01)$.

\begin{tabular}{cccc}
\hline Tissue types & $\mathrm{N}$ & $\begin{array}{c}\text { negative } \\
- \text { or }+\end{array}$ & $\begin{array}{c}\text { positive } \\
++ \text { or }+++\end{array}$ \\
\hline Normal bile duct tissue & $20(\%)$ & $15(75)$ & $5(25)$ \\
Stone + intrahepatic cholangiocarcinoma & $20(\%)$ & $5(25)$ & $15(75)$ \\
\hline
\end{tabular}

Table 5. Expression of Vimentin in normal bile duct tissues and bile duct carcinoma tissues $(\mathrm{P}<0.01)$.

\begin{tabular}{cccc}
\hline Tissue types & $\mathrm{N}$ & $\begin{array}{c}\text { negative } \\
- \text { or }+\end{array}$ & $\begin{array}{c}\text { positive } \\
++ \text { or }+++\end{array}$ \\
\hline Normal bile duct tissue & $20(\%)$ & $13(65)$ & $7(35)$ \\
Intrahepatic cholangiocarcinoma & $20(\%)$ & $4(20)$ & $16(80)$ \\
\hline
\end{tabular}

\subsection{Vimentin and the Expression of Intrahepatic Bile Duct Stones + Intrahepatic Cholangiocarcinoma}

Vimentin expression (normal bile duct tissue see Figure 7(a) and intrahepatic bile duct stones + intrahepatic bile duct carcinoma see Figure $7(\mathrm{~b})$ ), resulting in elevated Vimentin expression.

Immunohistochemical results showed as shown in Figure 7, the positive expression of N-cadherin in intrahepatic bile duct stones and intrahepatic bile duct carcinoma tissues was 16 cases, accounting for $80 \%$ of the total, while in normal bile duct tissues, there were 4 cases, accounting for $20 \%$ of the total. The positive expression of $\mathrm{N}$-cadherin in intrahepatic bile duct stones and intrahepatic bile duct carcinoma tissues was significantly up-regulated. See Table $6(\mathrm{P}<0.01)$.

\subsection{Twist1, Twist2 and the Expression of Intrahepatic Cholangiocarcinoma Cells and Intrahepatolithiasis + Intrahepatic Cholangiocarcinoma}

The expression of Twisit1 see (Figure 8(b) + Figure 8(c)) and Twisit2 see Figure 8(d) + Figure 8(e) (normal bile duct tissues (see Figure 8(a)) and intrahepatic bile duct cancer cells and intrahepatic bile duct stones + intrahepatic bile duct cancer cells) showed no difference in Twisit1 and Twisit2 $(\mathrm{P}>0.05)$.

\section{Discusssion}

In recent years, it is crucial to find molecular markers and prognostic indicators for the early diagnosis of cholangiocarcinoma, improve the ability of early diagnosis and surgical resection rate, and further clarify the molecular mechanisms related to the invasion and metastasis of cholangiocarcinoma, so as to find effective therapeutic targets for improving the overall therapeutic effect of cholangiocarcinoma patients. The development, invasion, and metastasis of malignant tumors is a sequential, multistep process that includes local invasion, invascular 


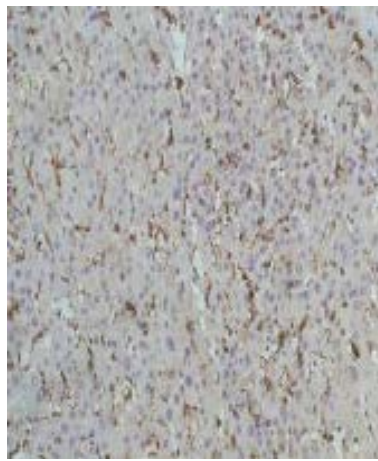

(a)

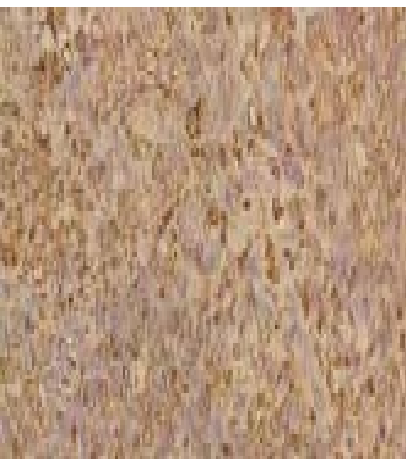

(b)

Figure 7. (a) Normal bile duct tissue; (b) Stone + intrahepatic cholangiocarcinoma.

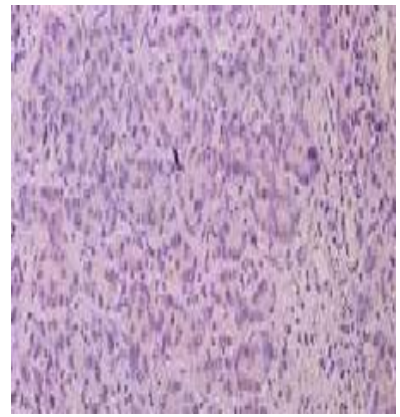

(a)

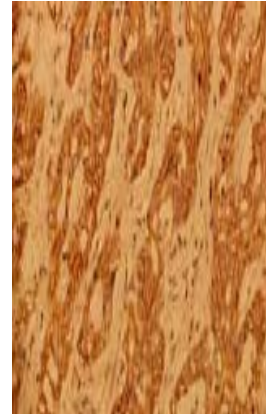

(b)

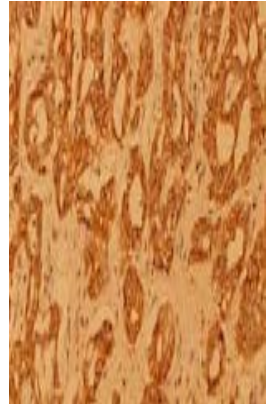

(d)

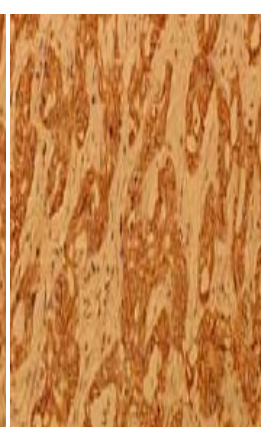

(c)

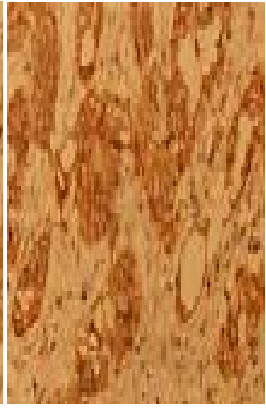

(e)

Figure 8. (a) Normal bile duct tissue; (b) Intrahepatic cholangiocarcinoma; (c) Stone + intrahepatic cholangiocarcinoma; (d) Intrahepatic cholangiocarcinoma; (e) Stone + intrahepatic cholangiocarcinoma. Twisit1 (b, c), Twisit2 (d, e). 
Table 6. The expression of Vimentin in normal bile duct tissue, intrahepatic bile duct stones and intrahepatic bile duct carcinoma $(\mathrm{P}<0.01)$.

\begin{tabular}{cccc}
\hline Tissue types & N & $\begin{array}{c}\text { negative } \\
- \text { or }+\end{array}$ & $\begin{array}{c}\text { positive } \\
++ \text { or }+++\end{array}$ \\
\hline Normal bile duct tissue & $20(\%)$ & $16(80)$ & $4(20)$ \\
Stone + intrahepatic cholangiocarcinoma & $20(\%)$ & $4(20)$ & $16(80)$ \\
\hline
\end{tabular}

penetration, stagnation and primary survival of distant targets, and finally the formation and colonization of micrometastases. In recent years, more and more studies have confirmed that epithelial-mesenchymal transformation is the key mechanism of the occurrence and development of various tumors, including cholangiocarcinoma, and plays an important role in invasion and metastasis [9]. Recently, epithelial-mesenchymal transformation has become an increasingly popular research direction to explain the mechanism of tumor metastasis and invasion. Ectomesenchymal into traditional theory of epithelial is divided into three type, and the associated with tumor invasion and metastasis of ectomesenchymal into 3 type of epithelium is a nonpolar connected to the basement membrane of epithelial cells in a series of regulation and control mechanism under the action of the corresponding change, loss of epithelial cell polarity characteristics, into a mesenchymal phenotype characteristics of cells capable of movement. Epithelial cells and mesenchymal cells exist in all organs of the human body, and each has its own characteristics: epithelial cells have polarity and close connections between cells, and their mobility and activity are poor. The mesenchymal cells do not have the polarity and tight intercellular junctions of epithelial cells and thus gain mobility. When tumor cells from epithelial cells after mesenchymal phenotype and function of cytoskeleton remodeling occurs, the adhesion between cells and cell loss and the activity of the cells and protein hydrolysis ability increase, thus gain the ability to break through the extracellular matrix and basement membrane, in interaction with neighboring cells and matrix and after immune escape, Finally, new colonization is formed in distant organ parts, and tumor invasion and metastasis are realized [10] [11].

Epithelial-mesenchymal transformation is therefore an important step in tumor invasion and metastasis. Furthermore, EMT is a process required for early metastasis of tumor cells by the combined action of inhibition of expression of genes that maintain epithelial characteristics and activation of mesenchymal phenotypes. EMT mechanism mediated process of invasion and metastasis of tumor cell proliferation regulation by various factors, has the characteristics of the differentiation, in solid carcinoma cells follow four steps [12]: 1) Through the connections between cells is reduced, the rearrangement of the cytoskeleton structure, with movement of phenotypic cell layer separation from the highly structured; 2) Isolated cells destroy the integrity of the basement membrane through active proteolysis, and then invade the surrounding stroma in a specific form. Once in the tumor stroma, cells can effectively interact with a variety of 
stromal and inflammatory cell types, further enhancing their invasive properties; 3) Tumor cells infiltrate into new vasculature with the help of local tumor microenvironment, and then spread far away through lymphatic and/or circulatory systems; 4) By moving from blood vessels to distant ectopic tissue parenchyma and colonization, the cells restart the uncontrolled proliferation program.

\section{Conclusion}

Epithelial-mesenchymal transformation of bile duct carcinoma can make cells lose the characteristics of epithelial cells. In this study, the expression of epithelial phenotypic marker E-cadherin protein decreased or even disappeared, the expression of intracellular skeleton protein molecular keratin decreased, and the expression of characteristic markers $\mathrm{N}$-cadherin and Vimentin of mesenchymal cells were up-regulated. Most of these EMT molecular markers are associated with tumor progression and poorer prognosis [13] [14]. We believe that these EMT-related phenotypic changes may be caused by the co-regulation of autocrine and paracrine signals released in the tumor microenvironment, including cytokines, growth factors, EMT-related stimulators, oncogenes, tumor suppressor genes and regulatory factors, and these signals have different associations with hypoxia and chronic inflammation. The EMT-like changes of bile duct carcinoma were induced jointly. In this study, the positive expression of Twist 1 and Twist2 in tumor cells of patients with cholangiocarcinoma was unrelated to the clinical data and prognosis of tumor embolism, lymph node metastasis, depth of tumor invasion, degree of tumor differentiation, and disease-free survival of patients. The expression of Twist1 and Twist 2 was consistent in tumor cells. Due to the small sample size, the influence of Twist 1 and Twist 2 on patients with cholangiocarcinoma still needs to be further explored.

\section{Foundation Project}

This study was supported by Hainan Provincial Basic (Project No. 819MS144).

\section{Conflicts of Interest}

The authors declare no conflicts of interest regarding the publication of this paper.

\section{References}

[1] Jemal, A., Bray, F., Center, M.M., et al. (2011) Global Cancer Statistics. CA: A Cancer Journal for Clinicians, 61, 69-90. https://doi.org/10.3322/caac.20107

[2] Halme, L., Arola, J., Numminen, K., et al. (2012) Biliary Dysplasia in Patients with Primary Sclerosing Cholangitis:additional Value of DNA Ploidity. Liver International, 32, 783-789. https://doi.org/10.1111/j.1478-3231.2011.02672.x

[3] Guglielmi, A., Ruzzenente, A., et al. (2014) Hepatolithiasis-Associated Cholangiocarcinoma:Results from a Multi-Institutional National Database on a Case Series of 23 Patients. European Journal of Surgical Oncology, 40, 567-575.

https://doi.org/10.1016/j.ejso.2013.12.006 
[4] Jusakul, A., Kongpetch, S. and Teh, B.T. (2015) Genetics of Opisthorchis Viverrini-Related Cholangiocarcinoma. Current Opinion in Gastroenterology, 31, 258-263. https://doi.org/10.1097/MOG.0000000000000162

[5] Lee, B.S., Park, E.C., Park, S.W., et al. (2015) Hepatitis B Virus Infection, Diabetes Mellitus, and Their Synergism for Cholangiocarcinoma Development: A Case-Control Study in Korea. World Journal of Gastroenterology, 21, 502-510. https://doi.org/10.3748/wig.v21.i2.502

[6] Shaib, Y.H., El-Serag, H.B., Davila, J.A., et al. (2005) Risk Factors of Intrahepatic Cholangiocarcinoma in the United States: A Case-Control Study. Gastroenterology, 128, 620-626. https://doi.org/10.1053/j.gastro.2004.12.048

[7] Fujita, T. (2013) Analyzing Risk Factors for Intrahepatic Cholangiocarcinoma. Hepatology, 58, 1862-1863. https://doi.org/10.1002/hep.26448

[8] Okuda, K., Nakanuma, Y. and Miyazaki, M. (2002) Cholangiocarcinoma: Recent Progress. Part 1: Epidemiology and Etiology. Journal of Gastroenterology and Hepatology, 17, 1049-1055. https://doi.org/10.1046/j.1440-1746.2002.02781.x

[9] Valastyan, S. and Weinberg, R.A. (2011) Tumor metastasis: Molecular Insights and Evolving Paradigms. Cell, 147, 275-292. https://doi.org/10.1016/j.cell.2011.09.024

[10] Kim, D., Xing, T., Yang, Z., et al. (2017) Epithelial Mesenchymal Transition in Embryonic Development, Tissue Repair and Cancer: A Comprehensive Overview. Journal of Clinical Medicine, 7, 25 p. https://doi.org/10.3390/jcm7010001

[11] Thiery, J.P. and Lim, C.T. (2013) Tumor Dissemination: An EMT Affair. Cancer Cell, 23, 272-273. https://doi.org/10.1016/j.ccr.2013.03.004

[12] Clark, A.G. and Vignjevic, D.M. (2015) Modes of Cancer Cell Invasion and the Role of the Microenvironment. Current Opinion in Cell Biology, 36, 13-22. https://doi.org/10.1016/j.ceb.2015.06.004

[13] Saentaweesuk, W., Araki, N., Vaeteewoottacharn, K., et al. (2018) Activation of Vimentin Is Critical to Promote a Metastatic Potential of Cholangiocarcinoma Cells. Oncology Research Featuring Preclinical and Clinical Cancer Therapeutics, 26, 605-616. https://doi.org/10.3727/096504017X15009778205068

[14] Gu, M.J. and Choi, J.H. (2014) Epithelial-Mesenchymal Transition Phenotypes Are Associated with Patientsurvival in Intrahepatic Cholangiocarcinoma. Journal of Clinical Pathology, 67, 229-234. https://doi.org/10.1136/jclinpath-2013-201806 